\title{
The Amygdala Central Nucleus and Appetitive Pavlovian Conditioning: Lesions Impair One Class of Conditioned Behavior
}

\author{
Michela Gallagher, ${ }^{1}$ Phillip W. Graham, ${ }^{1}$ and Peter C. Holland ${ }^{2}$ \\ 'Department of Psychology, University of North Carolina, Chapel Hill, North Carolina 27599, and ${ }^{2}+$ Department of \\ Psychology, Duke University, Durham, North Carolina 27706
}

\begin{abstract}
Rats with neurotoxic lesions of the amygdala central nucleus (CN) were trained using appetitive Pavlovian conditioning procedures. Conditioned responses (CRs) that are representative of 2 classes of behavior were monitored. One type of CR resembled the orienting responses that were elicited by the conditioned stimuli (CSs) prior to pairing with food reinforcement: the other type of CR resembled the behavior elicited by food reinforcement itself. Holland $(1977,1984)$ has referred to these as CS-generated and unconditioned stimulus (US)-generated CRs, respectively. During differential conditioning, some lesioned and unlesioned rats received reinforced presentations of a visual cue and nonreinforced presentations of an auditory cue, and the others in each lesion condition received reinforced auditory and nonreinforced visual cue presentations. Relative to the control group, the $\mathrm{CN}$-lesioned rats were impaired in the acquisition of CS-generated CRs to both visual and auditory CSs. Orienting responses and habituation to the CSs were, however, comparable for the lesion and control groups. Moreover, rats with CN lesions readily acquired the US-generated CRs. Thus, a specific class of conditioned behavior was impaired by $\mathrm{CN}$ damage. Many studies using aversive Pavlovian procedures have suggested that $\mathrm{CN}$ lesions impair fear conditioning. The present results suggest another role for $\mathrm{CN}$ in the conditioning of orienting/attentional responses.
\end{abstract}

Fifty years ago Kluver and Bucy (1939) observed that monkeys became remarkably tame after surgical removal of the temporal lobes, a phenomenon noted as early as 1888 by Brown and Schaefer. Much subsequent research extended these observations to suggest that the amygdala complex plays an important role in the expression of emotional behavior and in the association of stimuli with biologically significant events (Weiskrantz, 1956).

More recent research on the role of amygdala in simple forms of associative learning has focused on the central nucleus $(\mathrm{CN})$ and its descending projections to a variety of sensorimotor and visceromotor targets in the brain stem. Many investigations have shown the $\mathrm{CN}$ to be critical for the acquisition of defensive

\footnotetext{
Received Nov. 24, 1989; revised Jan. 8, 1990; accepted Jan. 9, 1990.

We wish to acknowledge the technical assistance of Marie Crock. This work was supported by NIMH grant MH35554 and a Research Scientist Development Award (KO2-MH00406) to M.G. and NSF grant 8513603 to P.C.H.

Correspondence should be addressed to Michela Gallagher, Ph.D., Department of Psychology, CB 3270, Davie Hall, University of North Carolina-Chapel Hill, Chapel Hill, NC 27599.

Copyright (c) 1990 Society for Neuroscience $0270-6474 / 90 / 061906-06 \$ 02.00 / 0$
}

Pavlovian conditioning, e.g., the conditioned potentiation of startle and autonomic conditioned responses (CRs) in rodents and rabbits (Gentile et al., 1986; Hitchcock and Davis, 1986; Kapp et al., 1979; Sananes and Campbell, 1989). Circumscribed damage to $\mathrm{CN}$ impairs the acquisition of these $\mathrm{CRs}$, and a variety of pharmacological manipulations in $\mathrm{CN}$ impair or facilitate this acquisition, depending on the agent used (Gallagher et al., 1980, 1981). Furthermore, electrophysiological studies of multiple- and single-unit activity indicate that conditioned neural responses emerge within the $\mathrm{CN}$ when animals are trained using defensive Pavlovian procedures (Applegate et al., 1982; Pascoe and Kapp, 1985). These findings support a common view that the $\mathrm{CN}$ is part of a system that regulates the acquisition of conditioned fear (Davis, 1986; Kapp et al., 1984), and is perhaps a site where the emotional significance of aversive events becomes associated with relevant conditioned stimuli (CSs).

The results of the experiment reported here suggest another role for amygdala $\mathrm{CN}$ in Pavlovian conditioning: the modulation of orienting/attentional responses to signals for biologically significant events. We used an appetitive conditioning preparation that permitted evaluation of the effects of $\mathrm{CN}$ lesions on 2 types of conditioned behavior in rats: (1) a conditioning-dependent enhancement of orienting responses elicited by the CSs prior to conditioning, so-called alpha conditioning (Hull, 1934), and (2) behaviors that were originally evoked by the food, unconditional stimulus (US), but not the CS. Considerable evidence (reviewed by Holland, 1984) indicates that these 2 classes of CRs involve different behavioral mechanisms. In the present experiment, rats with $\mathrm{CN}$ lesions showed substantial impairment in the acquisition of conditioned orienting responses but no deficit in acquisition of US-related behaviors.

The conditioning preparation employed here has been used extensively in studying behavioral aspects of Pavlovian conditioning to CSs paired with food (see Holland, 1977, 1984, 1989 , for reviews). Both visual and auditory CSs come to elicit behavior that resembles that elicited by food delivery itself: rats stand relatively motionless with their heads inserted into the recessed area where food is delivered and/or make short rapid movements of the head, directed toward the food cup. Because these behaviors are not evident during either modality cue prior to conditioning, and their form depends on the event used as the US (Holland, 1979a), they are described as "USgenerated" behaviors.

In addition to this class of CR, however, both CSs acquire high levels of behaviors that are elicited by those cues prior to conditioning: food-reinforced visual cues come to elicit rearing on the hind legs and orientation toward the light source (rear behavior) and the auditory cues acquire a startle response. Be- 
cause those behaviors depend on the nature of the CSs, are elicited by the CSs prior to training, and are little affected by the nature of the US (Holland, 1979a), they are described as "CS-generated" or "orienting" behaviors. The acquisition of these behaviors has been shown to be dependent on the Pavlovian (CS-US) relation: they quickly habituate if USs are not delivered, and unpaired presentation of USs produces relatively little sensitization of those responses (Holland, 1977). Furthermore, Holland (1979b, 1984) showed that accidental or "superstitious" operant contingencies contribute little to the emergence of these conditioned behaviors.

In the present experiment, these 2 classes of learned behavior were examined in a differential conditioning procedure that permitted comparison of the performance of lesioned and unlesioned rats during both reinforced $(\mathrm{CS}+)$ and nonreinforced $(\mathrm{CS}-$ ) presentations of visual and auditory CSs. Half of the subjects first received neurotoxic lesions of $\mathrm{CN}$, and the other half received only injections of a nontoxic vehicle into the $\mathrm{CN}$. Later, some of the rats in each lesion condition received rcinforced presentations of the visual cue and nonreinforced presentations of the auditory cue, and the others received reinforced auditory and nonreinforced visual cue presentations.

\section{Materials and Methods}

Subjects and surgery. Forty-five male Long-Evans rats (Charles River Laboratories, Raleigh, NC) were $300-325 \mathrm{gm}$ at the beginning of the experiment. Bilateral lesions of $\mathrm{CN}$ were made by injection of ibotenic acid (Sigma Chemical Company) while the rats were anesthetized with Nembutal $(50 \mathrm{mg} / \mathrm{kg})$. The stereotaxic coordinates used, with the head positioned level between bregma and lambda, were $-2.3 \mathrm{~mm}$ posterior to bregma, $\pm 4.2 \mathrm{~mm}$ lateral from the midline, and $-7.7 \mathrm{~mm}$ ventral from the skull surface. Ibotenic acid $(10 \mathrm{mg} / \mathrm{ml})$ was administered using a sterile PBS vehicle ( $\mathrm{pH} 7.4)$ in a volume of $0.3 \mu \mathrm{l} /$ site. After $7 \mathrm{~d}$ of postoperative recovery, rats were placed on a restricted feeding regimen. The rats were gradually brought down to $80 \%$ of their postoperative free-feeding weights and were maintained at those weights for the remainder of the experiment.

Apparatus. The apparatus consisted of 8 individual chambers, each $22.9 \times 20.3 \times 20.3 \mathrm{~cm}$, with aluminum front and back walls, clear acrylic sides and top, and a grid floor $(0.48 \mathrm{~cm}$ stainless steel rods spaced $1.9 \mathrm{~cm}$ apart). A dimly illuminated food cup was recessed in the center of one end wall. Each experimental chamber was enclosed in a soundresistant shell with acrylic windows for viewing the rats. A normallyoff $6 \mathrm{~W}$ houselight, which served as the visual $\mathrm{CS}$, and a speaker for delivering the auditory CS were mounted together on the inside wall of the shell, $25 \mathrm{~cm}$ above and behind the experimental chamber, even with the end wall that contained the food cup. Ventilation fans provided masking noise $(70 \mathrm{~dB})$, and a $6 \mathrm{~W}$ lamp behind a red lens provided dim background illumination. Two low-light television cameras were mounted $2.1 \mathrm{~m}$ from the experimental chambers so each could include 4 chambers in its field of view. Videocassette recorders were programmed to record behaviors that occurred during and $10 \mathrm{sec}$ before and after CS presentation.

Behavioral observation procedures. All observations were made from videotapes. Each rat's behavior was observed at $1.25 \mathrm{sec}$ intervals during the $5 \mathrm{sec}$ period immediately prior to CS presentations and during the CS presentations. In addition, the occurrence of a startle response (described later) during the first $1.25 \mathrm{sec}$ after CS onset was noted. All observations were paced by auditory signals recorded on the videotapes. At each observation, one and only one behavior was recorded.

Three behavioral categories are reported (see Holland, 1977, for a complete description). The 2 CS-generated responses reported were the rear, standing on the hind legs, with both front legs off the floor, but not grooming; and startle, a sudden gross body movement, often a jump, usually occurring at the onset of the auditory CS. The primary USgenerated behavior was standing motionless in front of the food cup with head or nose within the recessed area where food was to be delivered. This food cup category also included head jerk behavior: short, rapid horizontal and/or vertical movements of the head, typically oriented toward the food cup. Other behaviors, such as walking, grooming, or sitting quietly away from the food cup (see Holland, 1977), are not reported in this article. Figure 1 shows cartoons of the measures reported here: rear, startle, and food cup behaviors.

The measure of startle behavior, which invariably occurred during the first $1.25 \mathrm{sec}$ after onset of the auditory CS, was the percentage of trials on which that response occurred. The index of each of the other behaviors was percentage total behavior, obtained by dividing the frequency of the target behavior by the total number of observations made. Note that because the number of observations was constant within each observation interval, this measure is an absolute frequency measure, rather than a relative one. In addition, because rear behavior occurs mostly during the first $5 \mathrm{sec}$ interval of the $10 \mathrm{sec}$ visual cue, and food cup behavior during the latter 5 sec interval (Holland, 1977), we confined our reporting of those behaviors to those intervals. During $10 \mathrm{sec}$ auditory cues, however, food cup behavior is distributed relatively evenly in time (Holland, 1977), so we reported that measure over the entire $10 \mathrm{sec}$ interval. With both cues, however, statistical analyses of rear and food cup behavior from the entire $10 \mathrm{sec}$ interval or from either $5 \mathrm{sec}$ segment led to identical conclusions about the effects of $\mathrm{CN}$ lesions.

A single primary observer (P.C.H.) scored all of the behavioral data reported. To assess objectivity, a second observer also scored data from randomly selected sessions. The 2 observers agreed on $91 \%$ of 1256 joint observations. When the data were scored, neither observer was aware of the rats' lesion conditions.

Statistical analyses of all measures used 2-tailed distribution-free statistics. We adopted the $p<0.05$ level of significance.

Training procedures. The rats were first trained to eat from the food cups. Ten deliveries of two $45-\mathrm{mg}$ food pellets were given on a variabletime 2-min schedule in a single session. Next, the occurrence and habituation of CS-generated orienting behavior were examined. In each of two 60 -min pretraining sessions, four 10 -sec presentations of a 1500 $\mathrm{Hz}, 78 \mathrm{~dB}$ tone and four $10-\mathrm{sec}$ intermittent ( $3 \mathrm{~Hz}$ ) illuminations of the houselight were randomly intermixed, with intertrial intervals ranging from 4 to $12 \mathrm{~min}$ (mean, $7.5 \mathrm{~min}$ ).

The rats were then randomly (except for lesion condition) assigned to 4 groups. Group designations indicate whether the subjects had $\mathrm{CN}$ lesions (CN) or vehicle injections only (VE), and which CS (light or tone) was paired with the food US $(+)$. In each of 10 daily $60-\mathrm{min}$ conditioning sessions, all subjects received 4 presentations of the houselight (L) and 4 presentations of the tone $(\mathrm{T})$, randomly intermixed. In groups $\mathrm{CN} / \mathrm{L}+\mathrm{T}$ - and $\mathrm{VE} / \mathrm{L}+\mathrm{T}$-, each houselight presentation was followed immediately by the delivery of 2 food pellets, and the tone presentations were nonreinforced, whereas in groups $\mathrm{CN} / \mathrm{L}-\mathrm{T}+$ and $\mathrm{VE} / \mathrm{L}-\mathrm{T}+$, the tone presentations were reinforced and the houselight presentations were nonreinforced.

Histological procedures. After the completion of behavioral testing, all rats were deeply anesthetized with ether and perfused transcardially with $0.9 \%$ saline followed by a $10 \%$ formal-saline solution. Brains were removed and stored in $10 \%$ formalin for 1 week. Brains were then sectioned $(30 \mu \mathrm{m})$ on a freezing microtome, mounted on slides, and Nissl-stained. Histological examination was performed with the aid of the Paxinos and Watson atlas (1986) using an Olympus BH-2 microscope and computer-assisted video program (Bioquant) in order to record the location and quantify the size of the lesions. Histological determinations were made blind with respect to all behavioral data by one of the investigators (M.G.).

\section{Results}

Histological analysis. Histological analysis revealed that 14 of the 23 lesioned rats had neuron loss that was confined bilaterally to the $\mathrm{CN}$. The remaining animals in the lesion group had either unilateral $\mathrm{CN}$ damage (the contralateral injection site was located medial to $\mathrm{CN}, n=3$ ) or included damage outside of $\mathrm{CN}$ (anterior amygdala area or lateral/basolateral nuclei). Only animals with bilateral damage confined to the $\mathrm{CN}$ were used in the data analysis. The lesions ranged in size from $30-87 \%$ of the total area of $\mathrm{CN}$, with the average lesion encompassing approximately $70 \%$ of the nucleus. In all cases, the lesion sites were marked by heavy gliosis, and, at higher magnification, intact neurons were clearly visible at the borders of the lesions (see Fig. 2). In the vehicle group, injector tracts were visible in all cases. Six animals in this group were excluded because the 
Figure 1. Illustration of the types of behaviors observed during appetitive Pavlovian conditioning. After pairing the visual CS with food, a rear response is elicited by the houselight. This behavior resembles the orienting response to this visual cue. The auditory CS, in contrast, elicits a startle behavior which resembles the orienting response evoked by this cue prior to conditioning. Both visual and auditory CSs also elicit behavior that is directed toward the food cup prior to the delivery of the US (food). Unlike rear and startle CRs, food cup behavior is not elicited by the CSs prior to conditioning but depends on the event used as the US.

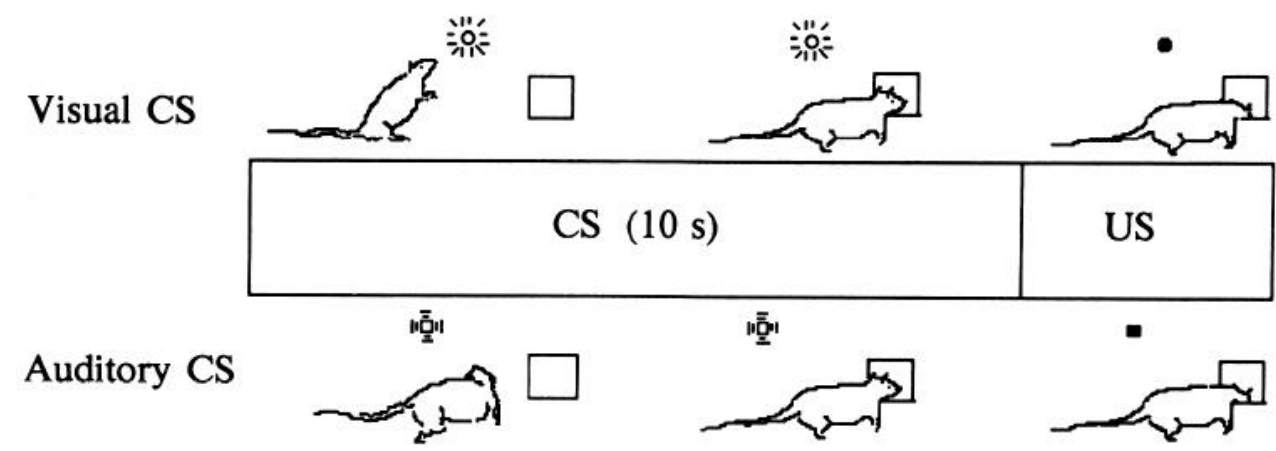

constituted more than $5 \%$ (each) of the total pre-CS behavior, and never differed reliably between groups. Consequently, preCS behavior is not described further.

Pretraining. Food cup behavior was not observed during the CSs in the pretraining phase. Orienting responses that did occur (rear and startle) are shown in Figure 3. The left side of Figure 3 shows unconditioned rear behavior during the houselight CS in the pretraining phase and during the first trial of the conditioning phase (before the first US was presented). Rearing occurred at high levels at the beginning of each session, but rapidly decreased in frequency with repeated trials. No reliable differences between lesioned and unlesioned rats were observed over all pretraining trials [Mann-Whitney $U(14,14)=65.5$ ], or on any individual trial $[U$ 's $(14,14) \geq 58]$. The right side of Figure 3 shows startle behavior during the tone CS. Startle responses decreased in frequency over trials. No reliable differences between lesioned and unlesioned rats were observed over all pretesting trials $[U(14,14)=65.5]$ or on any individual trial $\left[\chi^{2}\right.$ 's (1) $\leq 2.49$ ]. Thus, $\mathrm{CN}$ lesions had no significant effect on the initial elicitation of unconditioned orienting responses by CSs or on the habituation of those behaviors.

Conditioning. Although $\mathrm{CN}$ lesions had no effect on the unconditioned elicitation of the orienting responses, rear and startle, they severely impaired conditioning of those behaviors. Acquisition of rear behavior (Fig. 4, top-left panel) during the visual cue paired with food was substantial in unlesioned rats (group $\mathrm{VE} / \mathrm{L}+\mathrm{T}-$ ) but minimal in lesioned rats (group $\mathrm{CN} /$ $\mathrm{L}+\mathrm{T}-$ ). Over all 10 conditioning sessions, rear behavior of unlesioned rats during the visual cue was reliably more frequent when that cue was paired with food (group VE/L+T-) than when it was not paired with food [(group VE/L-T + ), $U(8,6)$ $=4.5$ ]. Conversely, rear behavior of lesioned rats was not reliably greater when the visual cue was reinforced with food (group $\mathrm{CN} / \mathrm{L}+\mathrm{T}-$ ) than when it was nonreinforced [(group $\mathrm{CN} /$ $\mathrm{L}-\mathrm{T}+), U(8,6)=10$ ]. Furthermore, rear behavior during the reinforced light was more frequent in unlesioned than lesioned rats $[U(8,6)=1]$, whereas the frequency of rear behavior during the nonreinforced light did not differ reliably $[U(8,6)=13]$. The apparent, albeit unreliable, difference in responding to the nonreinforced visual cue could merit further study but is unlikely to account for the magnitude of the conditioning impairment to the reinforced visual cue. Thus, the effects of the lesions on rear responding were largely confined to conditioning.

Although both lesioned and unlesioned rats acquired startle behavior to the tone paired with food (Fig. 4, top-right panel), that behavior was considerably more frequent in unlesioned rats. Over all sessions, reinforced tones elicited more startle
Figure 2. Lower panel, Diagram of the amygdala complex with the position of the photomicrograph (above) demarcated by the enclosed box and the area of the lesion identified by broken lines. Visible landmarks in the photomicrograph include the stria terminalis (at the left border) and intact darkly staining neurons in the basolateral nucleus ( $B L A$; near the border on the right). Heavy gliosis marks the site of the lesion. Spared neurons are evident in the far lateral $C N$. $C E$, central n.; $L a$, lateral n.; $M E$, medial n.; st, stria terminalis. 

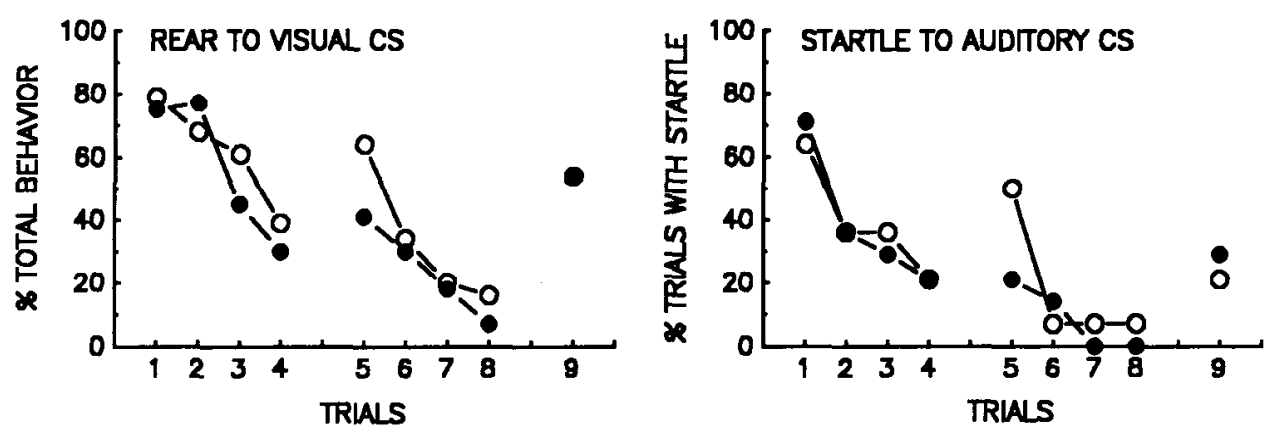

Figure 3. Orienting and habituation behavior elicited by the visual cue (left panel) and the auditory cue (right panel) during 2 sessions in the pretraining phase. The data points labeled 9 indicate responding to the initial presentation of the respective cues during the first conditioning session (prior to the first US pairing). Group $\mathrm{CN}$ had bilateral central nucleus lesions; group $\mathrm{VE}$ received only injections of vehicle into

GROUP VE

GROUP CN

$\mathrm{CN}$ during surgery. There was no significant effect of $\mathrm{CN}$ damage on either orienting or habituation.

behavior than nonreinforced tones in both lesioned (group $\mathrm{CN}$ / $\mathrm{L}-\mathrm{T}+$ vs group $\mathrm{CN} / \mathrm{L}+\mathrm{T}-$ ) and unlesioned (group VE/L- $\mathrm{T}+$ vs group $\mathrm{VE} / \mathrm{L}+\mathrm{T}-$ ) rats $[U \mathrm{~s}(8,6)=0$ ]. More important, unlesioned rats showed reliably more startle to the reinforced tone than lesioned rats $[U(8,6)=5.5]$, whereas startle responding clicitcd by nonreinforced toncs was similar in lesioned and unlesioned rats $[U(8,6)=21.5]$. Thus, the effects of the lesions on startle responding were confined to conditioning.

In contrast to their effects on CS-generated responses, the $\mathrm{CN}$ lesions had no reliable effect on the acquisition of the US-generated behaviors by either visual or auditory cues. Similar levels of behavior directed toward the food cup were acquired to the reinforced houselight CS (Fig. 4, bottom-left panel) in groups $\mathrm{VE} / \mathrm{L}+\mathrm{T}-$ and $\mathrm{CN} / \mathrm{L}+\mathrm{T}-[U(8,6)=11.5]$, and both of those groups showed more of this behavior than the 2 groups that received nonreinforced light presentations [(groups $\mathrm{VE} / \mathrm{L}-\mathrm{T}+$ and $\mathrm{CN} / \mathrm{L}-\mathrm{T}+), U \mathrm{~s}(8,6)=0$ ]. Likewise, similar levels of food cup behavior were acquired to the reinforced tone CS (Fig. 4 , bottom-right panel) in groups $\mathrm{VE} / \mathrm{L}-\mathrm{T}+$ and $\mathrm{CN} / \mathrm{L}-\mathrm{T}+[U(8$, $6)=13$, and both of those groups showed more of this behavior than the 2 groups that received nonreinforced tone presentations $[U ' s(8,6)=0]$.
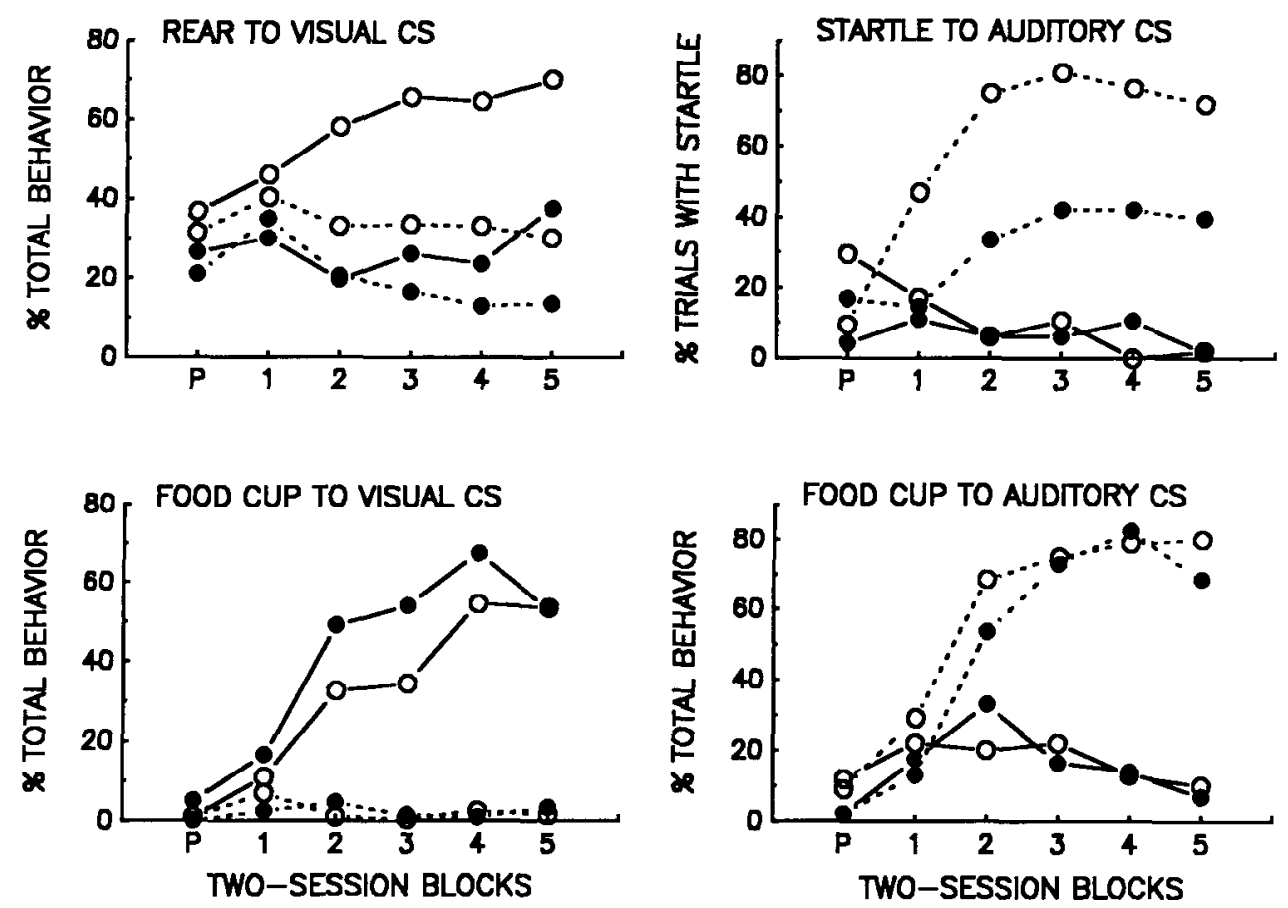

Figure 4. CS-generated (upper panels) and US-generated (lower panels) behaviors in the conditioning phase. The points labeled $P$ refer to mean responding during the last session of the pretraining phase. Group $\mathrm{CN}$ had bilateral central nucleus lesions; group VE received only injections of vehicle into $\mathrm{CN}$ during surgery. In the $\mathrm{L}+\mathrm{T}-$ training condition, the visual cue was reinforced but the auditory cue was nonreinforced. In the $\mathbf{L}-\mathrm{T}+$ condition, animals received reinforced auditory and nonreinforced visual cue presentations. See text for description of results and statistical analyses. 


\section{Discussion}

$\mathrm{CN}$ lesions impaired the acquisition of CS-generated CRs that resembled the original orienting responses. However, those lesions did not alter the unconditioned elicitation or habituation of the orienting/alerting behaviors in the pretraining sessions. Accordingly, the impaired acquisition of CS-generated CRs cannot be attributed to a loss of sensorimotor capacity. Nor can motivational or general learning deficits account for the effects of CN damage, because rats with lesions readily acquired USgenerated CRs. Thus, the effect of the CN lesions was limited to impairment of conditioning-dependent enhancement of orienting/alerting behavior.

These results are supported by the results of another experiment (Gallagher et al., 1989) that was designed to investigate the effects of small $\mathrm{CN}$ lesions on complex discrimination learning. That cxpcriment involved nonreinforced pretraining and conditioning with a visual cue and several auditory cues. Eight lesioned and eight unlesioned rats all received nonreinforced pretraining with a visual stimulus and 3 auditory stimuli, followed by reinforced training of one of the auditory cues and of a serial-trace compound CS, which comprised a visual cue followed by an empty trace interval and another auditory cue. As in the present experiment, CN lesions spared US-generated CRs but prevented acquisition of the conditioned orienting behavior. In addition, there was no detectable difference between the groups in unconditioned orienting behavior, including the initial level of orienting, habituation, or spontaneous recovery. In that experiment, there was also little evidence of conditioned orienting behavior to auditory CSs in the lesioned group. In the present study, the observation that $\mathrm{CN}$-lesioned animals showed some learning to the auditory $\mathrm{CS}$, but not to the visual CS, might be attributable to the fact that the lesions in group $\mathrm{CN} / \mathrm{L}+\mathrm{T}-$ were on average larger than the lesions in group $\mathrm{CN} / \mathrm{T}+\mathrm{L}-$ (approximately 80 vs $60 \%$ destruction of the nucleus).

Our results suggest a possible reinterpretation of some of the effects of $\mathrm{CN}$ damage in defensive conditioning tasks. For example, the impairment of defensively conditioned heart rate responses in rabbits has often been attributed to a general deficit in fear conditioning (e.g., Kapp et al., 1984). However, in those studies, the heart rate response occurred to the CS prior to conditioning, and thus might be viewed as a CS-generated orienting response, like rear and startle behaviors in the present experiment. Notably, Kapp et al. (1979) found that CN damage impaired the development of conditioned heart rate responses without altering either initial heart rate orienting or habituation, analogous to our findings with rear and startle behaviors. Furthermore, $\mathrm{CN}$ lesions that impair heart rate CRs spare nictitating membrane (NM)/eyeblink CRs (Gentile et al., 1986), USgenerated responses which, like food cup behavior in the present experiment, do not occur to the CS prior to conditioning. Although neurotoxic damage of the dorsal amygdala, not confined to $\mathrm{CN}$, in rats impairs aversively conditioned autonomic and somatomotor responses (Iwata et al., 1986), the concept that $\mathrm{CN}$ integrity is necessary for normal fear conditioning might deserve reconsideration: recent studies on the effects of discretc neurotoxic $\mathrm{CN}$ lesions have failed to reproduce the severe avoidance conditioning impairments found with electrolytic lesions (Jellestad et al., 1986; Riolobos and Garcia, 1987).

The present results and a number of previous observations are consistent with a view that $\mathrm{CN}$ is part of a system that determines the extent to which animals learn to monitor or altend to informative events, irrespective of the nature of the US. This view coincides with a growing appreciation for the neural circuitry that the $\mathrm{CN}$ shares with other structures in the basal forebrain (de Olmos et al., 1985; Alheid and Heimer, 1988).

The efferent projections of the $\mathrm{CN}$ gain access to hypothalamus and a number of brain-stem regions, e.g., somatomotor systems in the reticular formation, and autonomic areas. 'I hese targets include the parabrachial nuclei, the midbrain and pontine central gray, the dorsal motor nucleus of the vagus, and the nucleus of the solitary tract. Many of these targets are also reciprocally connected with CN (see Amaral, 1987, for a review).

There is, in addition, compelling evidence that the $\mathrm{CN}$ shares many of the aforementioned pathways, and is richly interconnected, with a number of regions that extend rostrally through the basal forebrain, e.g., the dorsolateral sublenticular substantia innominata, the lateral portion of the bed nucleus of the stria terminalis, and the medial nucleus accumbens (Alheid and Heimer, 1988). This system, including the $C N$, is further characterized by its access to the magnocellular neurons of the basal forebrain that innervate cortex (Price and Amaral, 1981; Groves and Nauta, 1984; Russchen et al., 1985). Thus, it may be useful to consider the $\mathrm{CN}$ as part of a more extensive basal forebrain system which includes both projections to brain-stem circuitry, where orienting reflexes may be largely organized, as well as access to cortical systems that may further regulate attentional processes. Indeed, alerting behavior elicited by low-level stimulation of the amygdala, first noted by Kaada (see Kaada, 1972. for review of earlier work), is found following microstimulation of the $\mathrm{CN}$ and occurs in conjunction with both autonomic responses and desynchronization of the cortical EEG (Stock et al., 1981; Kapp et al., 1982; Iwata et al., 1987).

Finally, the results reported herc complement Holland's contention that the conditioning of CS- and US-generated behaviors may involve different behavioral mechanisms, by raising the possibility that distinct neural mechanisms underlie the acquisition of those 2 classes of CRs (Holland, 1977, 1984). The extensive prior behavioral analysis of the conditioning preparation described in this report makes it an important resource for neurobiological analyses of learning of component responses and integration among behavioral systems.

\section{References}

Alheid GF, Heimer L (1988) New perspectives in basal forebrain organization of special relevance for neuropsychiatric disorders: the striopallidal, amygdaloid, and corticopetal components of substantia innominata. Neuroscience 27:1-39.

Amaral DG (1987) Memory: anatomical organization of candidate brain regions. In: Handbook of physiology, Sect 1 , the nervous system, vol 5, (Mountcastle VD, Plum F, Geiger SR, eds), pp 211-294. Bethesda, MD: American Physiological Society.

Applegate CD, Frysinger RC, Kapp BS, Gallagher M (1982) Multiple unit activity recorded from amygdala central nucleus during Pavlovian heart rate conditioning. Brain Res 238:457-462.

Brown S, Schaefer EA (1888) An investigation into the function of the occipital and temporal lobe of the monkey's brain. Phil Trans $R$ Soc Lond [Biol] 179:303-327.

Davis M (1986) Pharmacological and anatomical analysis of fear conditioning using the fear-potentiated startle paradigm. Behav Neurosci 100:814-824.

de Olmos J, Alheid GF, Beltramino CA (1985) Amygdala. In: The rat nervous system (Paxinos G, ed), pp 223-234. Sydney: Academic Press.

Gallagher M, Kapp, BS, Frysinger RC, and Rapp PR (1980) B-Adrenergic manipulation in amygdala central $n$. alters rabbit heart rate conditioning. Pharmacol Biochem Behav 12:419-426.

Gallagher M, Kapp BS, McNall CL, Pascoe JP (1981) Opiate effects 
in the amygdala central nucleus on heart rate conditioning in rabbits. Pharmacol Biochem Behav 14:497-505.

Gallagher M, Graham PW, IIolland PC (1989) The amygdala central nucleus and appetitive Pavlovian conditioning: lesions impair one class of conditioned behavior. Soc Neurosci Abstr 15:891.

Gentile CG, Jarrel TW, Teich A, McCabe PM, Schneiderman N (1986) The role of the amygdaloid central nucleus in the retention of differential Pavlovian conditioning of bradycardia in rabbits. Behav Brain Res 20:263-273.

Groves EA, Nauta WJH (1984) Light microscopic evidence for striatal and amygdaloid input to cholinergic cell group $\mathrm{CH} 4$ in the rat. Soc Neurosci Abstr 10:7.

Hitchcock J, Davis M (1986) Lesions of the amygdala, but not the cerebellum or red nucleus, block conditioned fear as measured with the potentiated startle paradigm. Behav Neurosci 100:12-22.

Holland PC (1977) Conditioned stimulus as a determinant of the form of the Pavlovian conditioned response. J Exp Psychol 3:77-104.

Holland PC (1979a) Differential effects of omission contingencies on various components of Pavlovian appetitive conditioned behavior in rats. J Exp Psychol: Anim Behav Proc 5:178-193.

Holland PC (1979b) The effects of qualitative and quantitative variations in the US on individual components of Pavlovian appetitive behavior in rats. Anim Learn Behav 7:424-432.

Holland PC (1984) Origins of behavior in Pavlovian conditioning. Psychol Learn Motiv 18:129-174.

Holland PC (1989) Forms of memory in Pavlovian conditioning. In: Brain organization and memory: cells, systems and circuits (McGaugh JL, Weinberger NM, Lynch G, eds), New York: Oxford U.P.

Hull (1934) Learning: II. The factor of the conditioned reflex. In: A handbook of general experimental psychology (Murchison C, ed), pp 382-455. Worcester, MA: Clark U.

Iwata J, LeDoux JE, Meeley MP, Arneric S, Reis DJ (1986) Intrinsic neurons in the amygdaloid field projected to by the medial geniculate body mediate emotional responses conditioned to acoustic stimuli. Brain Res 383:195-214.

Iwata J, Chida K, LeDoux JE (1987) Cardiovascular responses elicited by stimulation of neurons in the central amygdoid nucleus in awake but not anesthetized rats resemble conditioned emotional responses. Brain Res 418:183-188.

Jellestad FK, Markowska A, Bakke HK, Walther B (1986) Behavioral effects after ibotenic acid, 6-OHDA and electrolytic lesions in the central amygdala nucleus of the rat. Physiol Rehav 37:855-862.
Kaada BR (1972) Stimulation and regional ablation of the amygdaloid complex with reference to functional representations. In: The neurobiology of the amygdala (Eleftheriou BE, ed) pp 205-281. New York: Plenum.

Kapp BS, Frysinger RC, Gallagher M, Haselton JB (1979) Amygdala central nucleus lesions: effect on heart rate conditioning in the rabbit. Physiol Behav 23:1109-1117.

Kapp BS, Gallagher M, Underwood MD, McNall C, Whitehorn D (1982) Cardiovascular responses elicited by electrical stimulation of the amygdala central nucleus in the rabbit. Brain Res 234:251-262.

Kapp BS, Pascoe JP, Bixler MA (1984) The amygdala: a neuroanatomical systems approach to its contribution to aversive conditioning. In: The neuropsychology of memory (Squire L, Butters N, eds), pp 473-488. New York: Guilford Press.

Kluver H, Bucy PC (1939) Preliminary analysis of the temporal lobes in monkeys. Arch Neurol Psychiatry 42:979-1000.

Pascoc JP, Kapp BS (1985) Electrophysiological characteristics of amygdaloid central nucleus neurons during Pavlovian fear conditioning in the rabbit. Behav Brain Res 16:117-133.

Paxinos G, Watson C (1986) The rat brain in stereotaxic coordinates. New York: Academic.

Price JL, Amaral DG (1981) An autoradiographic study of the projections of the central nucleus of the monkey amygdala. J Neurosci 1:1242-1259.

Riolobos AS, Garcia AIM (1987) Open field activity and passive avoidance responses in rats after lesion of the central amygdaloid nucleus by electrocoagulation and ibotenic acid. Physiol Behav 39: $715-720$

Russchen FT, Amaral DG, Price JL (1985) The afferent connections of the substantia innominata in the monkey, Macaca fascicularis. $J$ Comp Neurol 242:1-27.

Sananes CB, Campbell BA (1989) Role of the central nucleus of the amygdala in olfactory heart rate conditioning. Behav Neurosci 103: 519-525.

Stock G, Rupprecht U, Stumpf H, Schlor KH (1981) Cardiovascular changes during arousal elicited by stimulation of the amygdala, hypothalamus and locus coeruleus. J Autonom Nerv Sys 3:503-510.

Weiskrantz L (1956) Behavioral changes associated with ablations of the amygdaloid complex in monkeys. I Comp Physiol Psychol 49: 381-391. 